\title{
The computer simulation of wear behavior appearing in total hip prosthesis ${ }^{\text {is }}$
}

\author{
James Shih-Shyn $\mathrm{Wu}^{\mathrm{a}, *}$, Jui-Pin Hung ${ }^{\mathrm{a}}$, Chi-Shiang Shu ${ }^{\mathrm{b}}$, Jian-Horng Chen ${ }^{\mathrm{c}}$ \\ a Institute of Mechanical Engineering, National Chung-Hsing University, 250, Kuo-Kuang Road, Taichung, Taiwan 402, Taiwan, \\ $R O C$ \\ ${ }^{\mathrm{b}}$ Advanced Semiconductor Engineering Inc., Kaohsiung, Taiwan 804, Taiwan, ROC \\ ${ }^{\mathrm{c}}$ Chung Shan Medical University, 110, Sec. 1, Chien-Kuo N. Road, Taichung, Taiwan 402, Taiwan, ROC
}

Received 20 April 2001; received in revised form 12 October 2001; accepted 12 November 2001

\begin{abstract}
Computer algorithms are proposed for the estimation of wear appearing in artificial hip joints using finite element analysis based on the modified Archard's wear law, contact features and an analogue wear process. A pin-on-disk plate experiment is reconstructed to assess the efficiency and validity of the algorithms proposed here. Through the successful verification of wear depth and volume loss of the pin-on-disk plate as well as the artificial hip joint, the current algorithms provide significant agreement with experiments, clinical measurements and numerical calculations and are shown to be both valid and feasible. Further investigation into the effect of femoral heads with various sizes suggests that the larger femoral head may induce larger wear volume but gives a smaller wear depth and that wear depth and volume loss are apparently nonlinearly related to the femoral head diameter. It is shown that the current algorithms are useful and helpful in understanding wear behavior for alternative or new designs of artificial hip joints and even for other analogous structures. (C) 2002 Elsevier Science Ireland Ltd. All rights reserved.
\end{abstract}

Keywords: Finite element; Hip prosthesis; Wear

\section{Introduction}

The post-implanted behavior of stems in total hip anthroplasty is of practical importance in medical problems. Biological failure may lead to the loosening of the hip implanted components,

\footnotetext{
No benefits in any form have been received or will be received from a commercial party related directly or indirectly to the subject of this article.

* Corresponding author. Tel./fax: + 886-4-2850-485.

E-mail address: sswu@dragon.nchu.edu.tw (J.S.-S. Wu).
}

which contributes to osteolytic reactions in the bone tissue caused by wear debris [1-5]. Wear debris induced from the surface of the polyethylene acetabular cup is a major factor leading to such biological failure [6,7]. Friction occurs due to the opposite motion of components in an artificial hip joint and generates worn out material, which may result in the malfunction of the prosthesis after long-term use [8].

Generally, studies into the wear of a polyethylene acetabular cup can be classified into three categories; clinical investigations, wear ex- 
periments and mathematical analyses. In clinical investigation, Atkinson et al. [9] used the casting technique to measure wear of the retrieved polyethylene acetabular cup sliding against the stainless femoral head. Livermore [10], Kabo [11] and Hall [12] measured wear rates by radiographic and shadowgraph techniques, respectively. Recently, Chen and $\mathrm{Wu}[13]$ proposed a new threedimensional methodology to evaluate the penetration of the femoral head by follow-up anteroposterior radiography. In the wear experiment, various apparatus, such as pin-on-disk/plate or hip simulators, have been designed to perform tests under various loading or lubricating conditions [14-16]. In their tests, ultra high molecular weight polyethylene (UHMWPE) material was adopted.

In the above two categories, clinical studies usually take a few retrieved hip joints for investigation and obtain some limited data for reference. In practice, wear depths and volume losses in the artificial joint are on three-dimensional behavior and it is difficult to obtain accurate results from a single two-dimensional X-ray film. Moreover, wear experiments are either time consuming or costly and the loading and boundary conditions applied to the specimens are often disputable.

For the purpose of imitating wear behavior appearing in a structure, mathematical analysis is practically an effective category. In this study, computer algorithms are proposed for the estimation of wear appearing in artificial hip joints using the finite element method.

\section{Background}

Computer simulation techniques are rapidly growing in their application in biomechanical fields. As a result of the high efficiency and reliable results obtained, studies concerning the estimation of structural wear have focused on the utilization of computer programs and numerical methods. In the past few years, Wang et al. [17], Pietrabissa et al. [18], Jin et al. [19] and Maxian et al. [20] used the Hertz theorem to calculate contact stresses on the interface and estimate the wear depth and the wear volume using the simplified
Archard's model in different forms. The Hertz contact theorem can be used only for contact bodies with conformal counterface (contact surface) under steady loadings or static loadings. However, the contact status between the hemispherical acetabular cup and the ball shaped femoral head must consider not only the variation of three-dimensional geometry, but also the difference in materials. Forces transmitted to the hip joint are quite complicated and stresses across the counterface between the acetabular cup and the femoral head appear discontinuous and may affect the contact status and wear behavior of the anthroplasty. Therefore, in order to simulate wear behaviors of a polyethylene acetabular cup accurately, we propose a modified Archard's wear formula and history-dependent processes in this study.

At the beginning of this paper, formulations of the wear mechanism and contact stresses are introduced. Finite element models of a pin-on-disk plate and an artificial hip joint are then constructed that include the appropriate loading and boundary conditions. Computational processes are given next in detail. Results related to wear depth and volume loss for either the pin-on-disk or the artificial hip joint are derived and compared with some other available experimental, clinical and numerical data. Finally, a summary is given.

\section{Design consideration}

\subsection{Model formulation}

\subsubsection{Wear mechanism}

Studies concerning wear behavior of UHMWPE sliding against stainless steel have been performed, for example Atkinson et al. [9], Wang et al. [17,21] and Wang and Li [22]. According to their descriptions, there are three periods existing in a sliding wear process: the wearing-in period, the steady-state period and the severe wear period. The shear of polyethylene particles on the interface in the wear-in period produces wear debris. In the steady-state period, the wear debris is produced from components of soft material and adheres to the contact surface of metal to generate 
a transfer film, which may result in the continuous formation of wear debris by adhesion. Severe wear, also called the fatigue wear, occurs when the contact stress exceeds the yielding strength of the material. It follows that a micro-crack is initiated on the subsurface and delaminating takes place under long-term repeated loadings. Generally, material wear in the first two periods may be ascribed to abrasion and adhesion, which are the two dominant mechanisms under normal loading conditions. This is also true as the polyethylene component slides against the smooth metal surface repeatedly. The main factors affecting wear behavior can be summarized as the contact status, the lubrication condition, material properties and the wear history. As for the wear rate, many models and equations based on the wear mechanism have been developed where the wear volumes for different mating components were derived [23]. Among these models, Archard's wear law refers to the combination of effects in the wear behavior and wear mechanism of the UHMWPE cup sliding against the femoral metal of the artificial hip joints. The amount of wear removed from the polyethylene cup can thus be assessed by the equation

$V=K \frac{S p_{\mathrm{n}}}{H}$

where $V$ is the linear wear volume removed from the soft material, $S$ is the sliding distance, $H$ is the hardness of the material, $p_{\mathrm{n}}$ is the normal contact force and $K$ is a proportional constant.

From the viewpoint of macroscopic wear mechanism, it can be seen from Eq. (1) that the wear volume correlates with material properties and moving activities only. Because components in the total hip joint prosthesis vary both in material properties and geometries, the contact status and wear phenomena must be considered as a threedimensional composite manner, which is historydependent. In addition, stresses and the wear depths on the contact surface at the femoral head are different from those at the polyethylene cup. So, the above equation cannot be directly applied to assess the wear volume for such a complicated structure. Thus, Archard's wear law must be modified into an incremental form as $d V=\Delta x \Delta y d h=K \frac{(\sigma d A) d S}{H}$

where $d V$ is the increment of wear volume, $d A=$ $\Delta x \Delta y$ is the infinitesimal contact area, $d h$ is the infinitesimal wear depth, $d S$ is the infinitesimal sliding distance and $\sigma$ is the normal contact stresses.

Eliminating $\Delta x \Delta y$ term from both sides of Eq. (2) gives

$d h=k_{\mathrm{w}} \cdot \sigma \cdot d S$

where $k_{\mathrm{w}}=K / H$ is the so-called wear coefficient in unit of $\mathrm{mm}^{3} / \mathrm{N}-\mathrm{m}$, which is a function of the material properties and the counterface roughness. The wear coefficient can be obtained from the wear experiment.

Integrating Eq. (3) along the sliding distance $S_{\mathrm{t}}$, the wear depth $h$ at any point on the interface can be obtained by

$h=\int_{s_{\mathrm{t}}} k_{\mathrm{w}} \cdot \sigma \cdot d S$

Eq. (4) can be written into the summation form at point $i$ as

$h_{i}=\sum k_{\mathrm{w}} \cdot \sigma_{i} \cdot d S$

The wear depth at any point on the counterface can thus be expressed in an alternative form for an isoparametric finite element interpolation as

$h_{i}=\sum N_{i} h_{i}$

where $N_{i}$ is the finite element interpolation function for node $i$. The corresponding wear volume removed from the contact surface $\Gamma$ is finally given by

$V=\int_{\Gamma}\left(\int_{S_{\mathrm{t}}} k_{\mathrm{w}} \cdot \sigma \cdot d S\right) \cdot d A$

\subsubsection{Contact stresses}

As described above, the contact status between the femoral head and the acetabular cup affects the wear behavior of the anthroplasty significantly. The wear depth and volume loss of the acetabular cup depend not only on the properties of materials, such as surface hardness, strength, 
friction property etc., but also on the contact status, such as contact region and corresponding stresses. Since a dynamic force acts on a body that is in contact with another, at any time stresses across the contact surface are discontinuous and the contact region is changed in a manner that is significant from the point of view of wear. So, in order to accurately calculate the wear depth and volume loss of the body, a finite element approach to solving such interface problems in a three-dimensional manner is formed next.

Considering two bodies in contact under static or steady loading conditions, the contact status can be described in the matrix form of

$$
\begin{aligned}
& {[\mathbf{K}]_{\mathrm{A}}\{\boldsymbol{q}\}_{\mathrm{A}}=\{\boldsymbol{F}\}_{\mathrm{A}}^{\text {ext }}+\{P\}_{\mathrm{A}}} \\
& {[\mathbf{K}]_{\mathrm{B}}\{\boldsymbol{q}\}_{\mathrm{B}}=\{\boldsymbol{F}\}_{\mathrm{B}}^{\text {ext }}+\{P\}_{\mathrm{B}}}
\end{aligned}
$$

where $\{\boldsymbol{F}\}$ specifies the external force vector, $[\mathbf{K}]$ is the general stiffness matrix of structures, $\{\boldsymbol{q}\}$ is the nodal displacement vector and $\{P\}$ is the induced contact force over the contact surface. Subscripts A and B denote structural characteristics for bodies A and B, respectively and superscript 'ext' denotes the external characteristics.

Generally, forces can be transmitted between independent bodies by fixed connectivity. So, the contact region between bodies $\mathrm{A}$ and $\mathrm{B}$ needs to be simulated with some virtual linkages, called contact elements [24] here, to overcome this difficulty. The stiffness $[\mathbf{K}]_{\mathrm{c}}$ for each contact element can be expressed, in the general finite element form, as

$[\mathbf{K}]_{\mathrm{C}}=\int_{S_{\mathrm{c}}}\{\mathbf{B}\}^{T}[\mathbf{D}]\{\mathbf{B}\} d S_{\mathrm{C}}$

where

$[\mathbf{D}]=\left[\begin{array}{ccc}k_{s 1} & 0 & 0 \\ 0 & k_{s 2} & 0 \\ 0 & 0 & k_{n}\end{array}\right]$

is the contact elasticity matrix. Coefficients $k_{s 1}$ and $k_{s 2}$ in [D] are the shear stiffness of elasticity along two orthogonal directions on the point of contact surface and $k_{\mathrm{n}}$ is the normal stiffness of elasticity on the point of contact surface. $[\mathbf{B}]$ is the stress-strain matrix of the contact element and $S_{\mathrm{c}}$ is the contact area.
Finally, assemblage of all element stiffness matrices may provide the equilibrium equation for the composite contact structure as

$[\mathbf{K}]\{\boldsymbol{q}\}=\{\boldsymbol{F}\}_{\mathrm{ext}}$

In the above $[\mathbf{K}]$ is the assemblage of $[\mathbf{K}]_{\mathrm{A}},[\mathbf{K}]_{\mathrm{B}}$ and $[\mathbf{K}]_{\mathrm{C}}$ described in Eq. (8) and Eq. (9) and $\{\boldsymbol{F}\}_{\text {ext }}$ is the vector of total external forces.

From results of Eq. (10), we may calculate stresses on the contact surface for either body. Then, substitution of such derived stresses into the wear formula may yield certain wear depths and volumes for either one.

\subsection{Finite element models}

\subsubsection{The pin-on-disk model}

In 1997, Saikko [25] studied wear rates and wear mechanism of retrieved polyethylene acetabular cups by experimental methods. In Saikko's investigation, a circularly translating pin-on-disk (CTPOD) sliding system was made. The cylindrical pin was made of ultra high molecular weight polyethylene. The length and the diameter of the pin were 9.5 and $9 \mathrm{~mm}$, respectively. The end of the pin was chamfered at an angle of $60^{\circ}$; therefore, the initial wear surface was flat with a diameter of $3.0 \mathrm{~mm}$. The disk-shaped plate, with outer diameter of $30 \mathrm{~mm}$, thickness of $10 \mathrm{~mm}$ and the surface roughness of $0.004-0.005 \mu \mathrm{m}$ was made of $316 \mathrm{~L}$ ASTM F318 annealed stainless steel. Material properties, such as young modulus $(E)$ and Poisson ratio ( $v$ ) were $E=0.8 \mathrm{GPa}, v=$ 0.47 for pin and $E=190 \mathrm{GPa}, v=0.305$ for the disk plate. Load applied to the top of pin was 70.7 N. In Saikko's experiment, the pin moved on the stiff plate along a circular route with a diameter of $10 \mathrm{~mm}$ and the sliding direction of pin was thus changed continuously.

In order to assess the validity of the wear simulations proposed here, we reconstruct a threedimensional pin-on-disk finite element model analogous to Saikko's CTPOD test. The model consists of three parts: pin, plate and pin holder, as shown in Fig. $1^{1}$. Again, contact elements are

\footnotetext{
${ }^{1}$ All shaded-images in this report are made from FEAST CAD system coded by the first author.
} 
added to imitate the interface between pin and the disk plate.

\subsubsection{The artificial hip joint model}

In the artificial hip joint model, the femoral head is made of stainless steel with an outer diameter of $22 \mathrm{~mm}$, the surface roughness is between 0.004 and $0.005 \mu \mathrm{m}$. The acetabular cup is made of UHMWPE with an interior diameter of $22 \mathrm{~mm}$ and an outer diameter of $50 \mathrm{~mm}$. The wear coefficient at the interface between hip joint and acetabular cup is $0.8 \times 10^{-6} \mathrm{~mm}^{3} / \mathrm{Nm}$, which is the same as that used by Saikko [25] and within

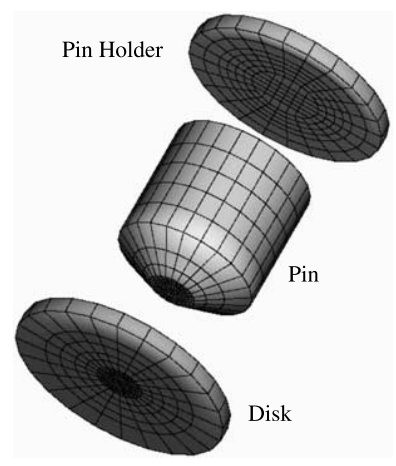

Fig. 1. Solid model with mesh overlay for the assessment of validity.

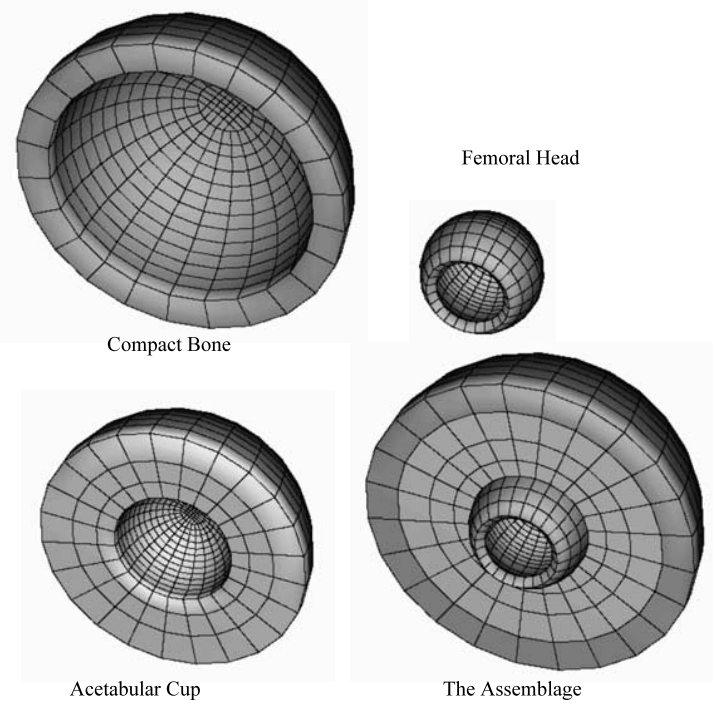

Fig. 2. Solid model with mesh overlay of artificial hip joint. the range declared in most studies $[9,26]$. Other material properties are described in Section 3.2.1. Here, the femoral head is simulated by a hollow spherical geometry with the interior surface constrained and meshed with 866 nodes and 420 hexahedron brick elements. The acetabular cup is divided into three layers meshed with 1444 nodes and 1044 hexahedron brick elements. At the possible contact regions ${ }^{2}$, such as bone/cup and cup/ head, the element discretization is meshed finely and the proper number of contact elements is added. Since the acetabular cup is fixed to the pelvis bony part, a layer of compact bone, with Young's modulus of $17 \mathrm{GPa}$ and Poisson ratio of 0.46 , is applied to the outer surface of the acetabular cup. A layer of compact bone is divided into 722 nodes and 348 hexahedron brick elements (Fig. 2).

The loading conditions depend on the activity of the hip joints. In fact, the kinematics of hip joints is quite complicated and difficult to describe in a mathematical way. From gait analyses, it can be observed that the main activities of the foot are flexible and extendable in the walking direction, while other activities, such as abduction/adduction and femoral rotation, are negligible [14]. Consequently, we only need to simulate the activity of flexion and extension of the foot during the normal gait. For actual walking activities, each gait consists of two phases: standing and swing phases. Therefore, forces acting on hip joints are varied in magnitude with time during the gait period and can be referred to a dynamic loading. Saikko [25] measured the load history for each gait cycle by a five-station hip joint simulator and declared the maximum force is $3.5 \mathrm{kN}$ and the swing angle is $23^{\circ}$ in the forward and backward directions for flexion/extension actions for each gait $[14,15]$. Here, we divide Saikko's gait cycle into 16 load stages and the force acting on the hip joint for each stage may be obtained (Fig. 3). The

\footnotetext{
${ }^{2}$ A pre-calculation for the combined model without contact elements under centrally distributed load gives the deformed shapes of the soft acetabular cup and the hard femoral head. The cup deformed concave in the central part and convex in the outer part. The line of inflection of the concave/convex areas tells the initial contact extent.
} 


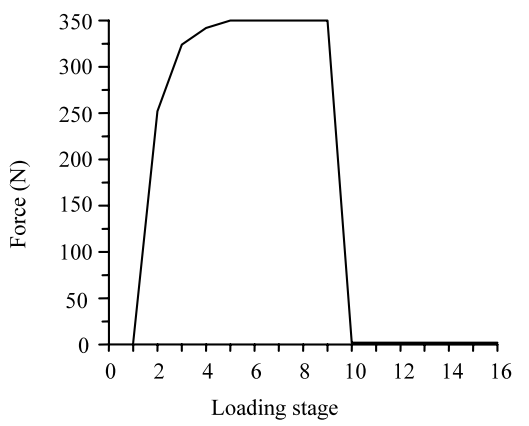

Fig. 3. Forces acting on the hip joint in one gait cycle.
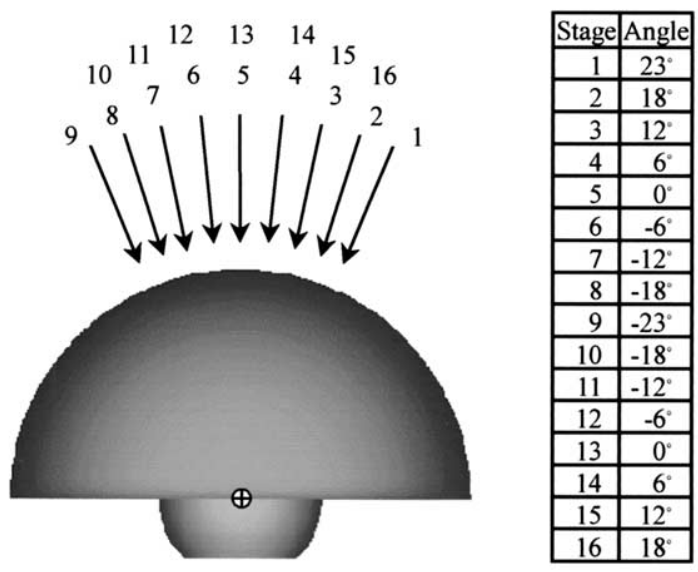

Fig. 4. Loading directions for 16 stages in one gait cycle.

direction of the force applied to the hip joint model depends on the swing direction of the femoral head. Fig. 4 shows the relative directions of these forces applied to the artificial hip joint. Thus, the loading pressure imposed on the model for each stage is calculated from the magnitude of the force obtained and the projected area of the outer surface of compact bone normal to the direction of the force. Some 1.5 million gait cycles were totally applied to the artificial hip joint to assess the wear depth/volume in this study [20].

\section{Computational processes}

As stated above, the material wear behavior is history-dependent. From the reality of the wear status, the mechanism involved in wear processes and the choice of appropriate wear model are critical. The magnitude of forces applied to the artificial hip joint in every stage of each gait cycle can thus lead to the real contact status. Therefore, it is necessary to change the extent of the counterface at the acetabular cup and the femoral head by incorporating wear depth and volume losses into the model geometry when wear occurs.

Based on the above considerations, the simulation process is considered and illustrated with a flow chart, as shown in Fig. 5. The system of the current algorithm is operated as an iterative process. Once reaching a designated gait cycle, the model geometry and the contact region are modified. The overall programs coded in FORTRAN with 64-bit data precision consist of two parts: three-dimensional contact stress analysis and wear assessment. For a three-dimensional contact structure with different material proper-

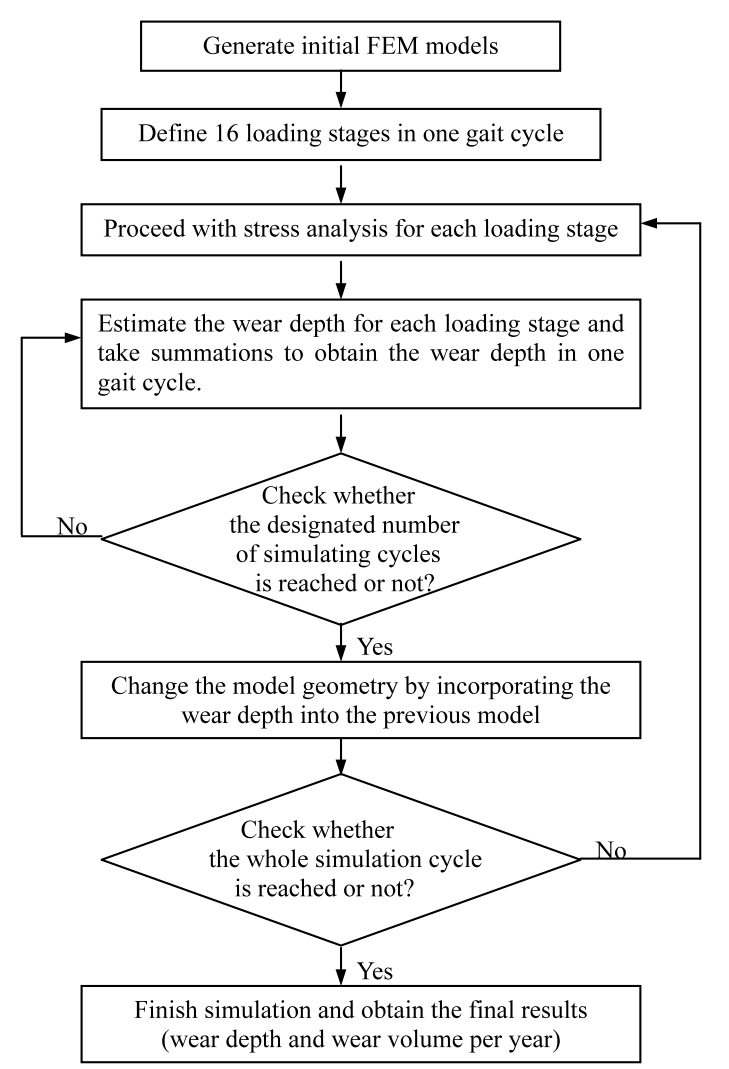

Fig. 5. Numerical procedures for wear estimation. 


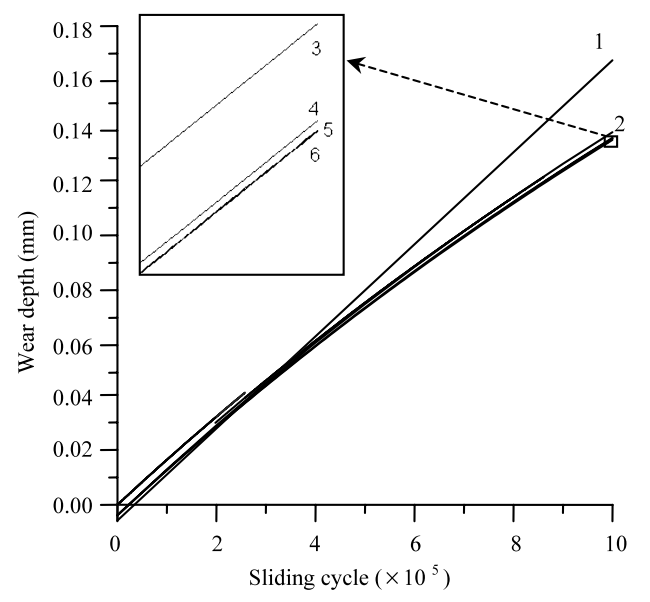

Fig. 6. Growth of wear depth of pin for six courses.

Table 1

Wear depths of pin-on-disk plate model for six courses

\begin{tabular}{lrl}
\hline Course & \multicolumn{1}{l}{$N^{*}$} & Wear depth $(\mathrm{mm})$ \\
\hline 1 & $1,000,000$ & 0.16756 \\
2 & 100,000 & 0.13946 \\
3 & 10,000 & 0.13738 \\
4 & 1000 & 0.13718 \\
5 & 100 & 0.13716 \\
6 & 10 & 0.13716 \\
\hline
\end{tabular}

* Designated number of model-modified cycles.

ties, the contact stresses on the interface at structures can thus be estimated and the wear depth and the wear volume can also be obtained by using the current algorithm.

\section{Results}

\subsection{Assessment of validity from the pin-on-disk model}

Based on Saikko's experiment [25], an analogous pin-on-disk plate model, under a force of 70.7 Newtons sliding for one million cycles, was made from hexahedron brick elements for the assessment of validity of the current algorithm.

In order to understand the effect of the model modification, we set up six courses with designated model-modified cycles, say, 1,000,000,
$100,000,10,000,1000,100$ and 10 , respectively. For each course, the wear simulation was completely performed and modification of the pin model was carried out after reaching the designated model-modified cycle. Results were obtained, as shown in Fig. 6, for the growth of wear depths and related data, as listed in Table 1. It can be seen that if the model modification is not performed during the simulation, i.e. in the case of course 1, the wear depth obtained equals $0.16756 \mathrm{~mm}$, which clearly differs from all the other values in the table. Once the model modified-cycle is increased, the wear depth will converge to $0.13716 \mathrm{~mm}$, which is in significant agreement with the wear depth of $0.13 \mathrm{~mm}$ for one million sliding cycles given by Saikko [25]. It indicates that the proper designated number of cycles to modify the wear model is necessary in the computer simulation. From our detailed inspection of the configurations of the pin in the wear processes, it was found that the wear depth of the pin is correlated closely with the contact area. It was also found that the maximum contact Von Mises stress derived by the current algorithm is equal to $3.8 \mathrm{MPa}$, which is well below the Von Mises yielding strength of $10.8 \mathrm{MPa}$ for a polymer pin [27]; therefore, plastic strains are hard to produce at the counterface and the occurrence of fatigue wear is impossible. These results encourage us to investigate further the wear of artificial hip joints.

\subsection{Wear analysis of the artificial hip joint}

According to the 16 loading stages for one gait cycle, we first calculated the normal pressures applied to the compact bony part for each load stage. Again, the contact elements were added to the counterfaces of the bone/cup and cup/head and 1.5 million gait cycles were executed. The initial extent of counterface between the acetabular cup and the femoral head was pre-calculated as before. Fig. 7 gives the shaded-image of wear depth on the counterface at the acetabular cup. The growth of wear depth and volume losses of the acetabular cups is presented for all gait cycles, as shown in Figs. 8 and 9, respectively. The wear depth and wear volume derived here are 0.111 


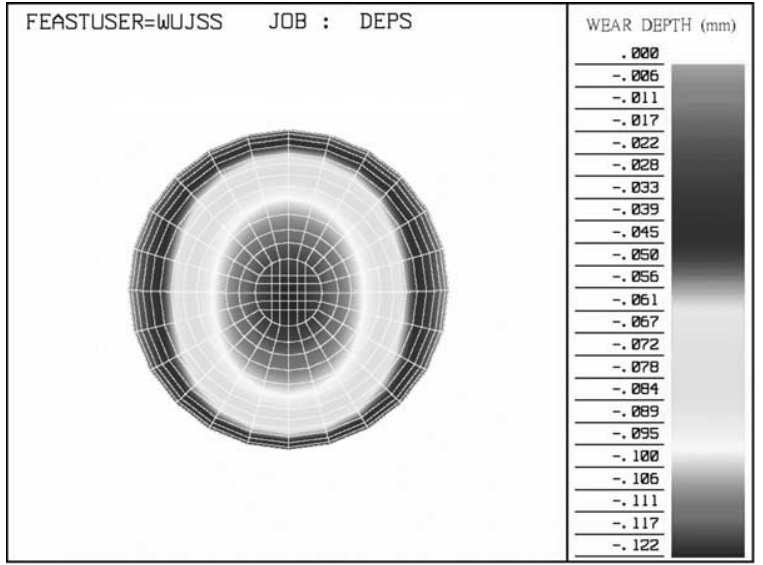

(a). No model modification

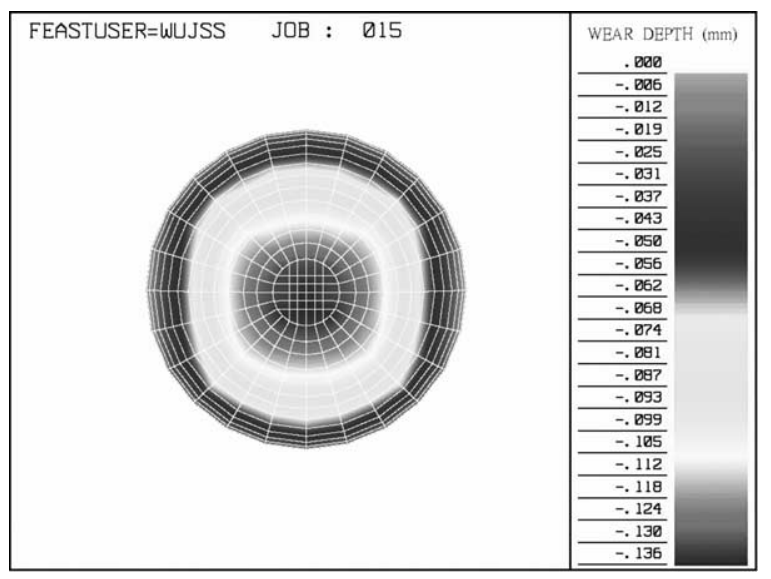

(b). With model modification

Fig. 7. Shaded-image of wear depth of acetabular cup.

mm per year and $42.0 \mathrm{~mm}^{3}$ per year, respectively. Livermore et al. [10] demonstrated a radiographically observed linear wear rate of $0.13 \mathrm{~mm}$ per year and a volumetric wear rate of $48 \mathrm{~mm}^{3}$ per year with the same size of femoral head. Saikko et al. [14] obtained a linear wear rate of $0.15 \mathrm{~mm}$ per year and a volumetric wear rate of $48.9-66.4 \mathrm{~mm}^{3}$ per year for the same size of femoral head by hip joint simulator. Other numerical results, such as those obtained by Maxian [20], also showed a linear wear rate of $0.11 \mathrm{~mm}$ per year, but with a volumetric wear rate of $13 \mathrm{~mm}^{3}$ per year. Note that Maxian's volumetric wear rate is much lower than all others referred to above. Comparison of wears depths and volume losses of the acetabular cup with a diameter of $22 \mathrm{~mm}$ obtained from clinical measurements, experimental studies and numerical methods is illustrated in Table 2. In the table, although some investigations explored a larger range of wear rates, it can be seen that the mean wear depth falls into the range of $0.10-0.15$ $\mathrm{mm}$ per year and the mean volumetric wear is in the range of $40-70 \mathrm{~mm}^{3}$ per year. Tables 3 and 4 illustrate wear depths and volume losses for the femoral head with diameters of 28 and $32 \mathrm{~mm}$, respectively. Again, the current algorithms may provide accurate results. It can be said that the computer simulation algorithm proposed here yields realistic results and is clearly feasible.
In the study, further explorations allow us to understand more about wear behavior in the artificial hip joint, corresponding to different sizes of femoral head, say, 22, 24, 26, 28, 30 and 32 $\mathrm{mm}$. Wear depths and volume losses on the acetabular cup for these femoral heads using current algorithms are derived and listed in Table 5. This demonstrates that the larger femoral head may induce larger wear volume, but results in a smaller wear depth. Curves fitted with nonlinear polynomials for wear depth and wear volume versus diameter of the femoral head are shown in Figs. 10 and 11, respectively. The magnitude

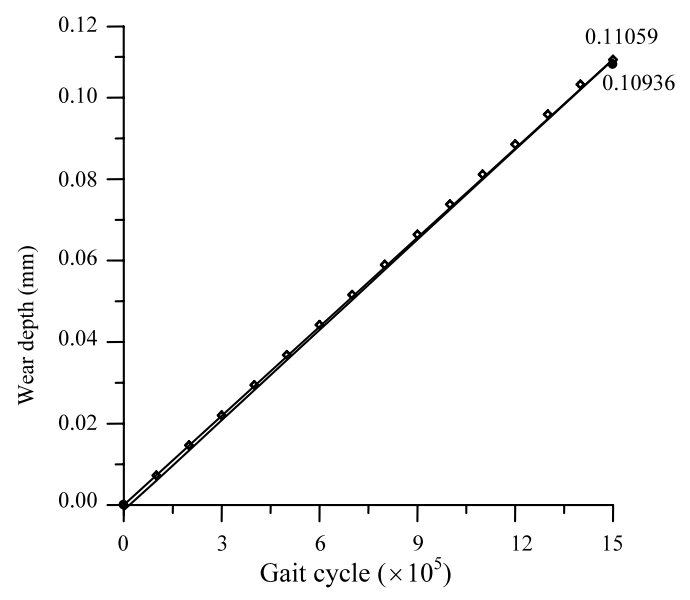

Fig. 8. Growth of wear depths of acetabular cup. 


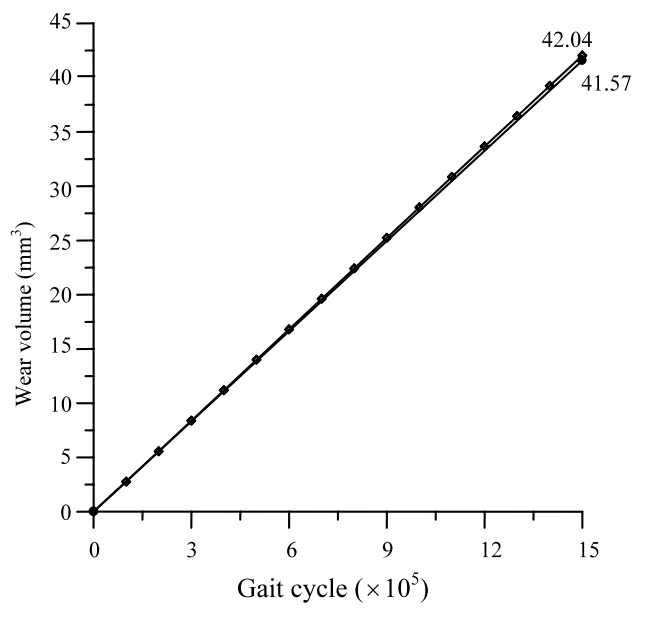

Fig. 9. Growth of wear volumes of acetabular cup.

of wear depth is obtained using the formula of $\left(0.298278-0.0117553 D+0.000147321 D^{2}\right)$, while the wear volume is obtained from ($1.42629+2.22132 D-0.0110269 D^{2}$ ), where $D$ is the diameter of the femoral head.

\section{Summary}

A finite element method is proposed for the estimation of wear appearing in artificial hip joints based on the modified Archard's wear law, contact features and a history-dependent process. Since the wear behavior and wear volume depend not only on the material properties, but also on the wear process, the contact surface between femoral head and acetabular cup is varied in a three-dimensional manner. Besides, a wear factor obtained from the experimental test on a specific hip joint is necessary. Due to the reality of physical phenomena of structural wear, the appropriate material/geometry nonlinear processes and wear mechanism have to be considered. Thus, a proper stage of modification on the wear model is necessary in the computational process. In this study, through the validity of pin-on-disk model, the result shows good agreement compared with the experiment data reported in literatures. For femoral head with a radius of $22 \mathrm{~mm}$, the average

\section{Table 2}

Comparison of wear depths/volumes of acetabular cup obtained from clinical measurements, experimental procedures and numerical methods

\begin{tabular}{llcc}
\hline Algorithm & Technique/model & Wear depth (mm/year) & Wear volume (mm ${ }^{3} /$ year) \\
\hline Clinical measurement & Casting technique [9] & $0.005-0.623$ (mean: 0.19) & $1.9-237$ (mean: 74) \\
& Radiograph technique [10] & $0-0.39$ (mean: 0.13) & $0-147$ (mean: 47.5) \\
& Radiograph technique [12] & $0.1-0.15$ & 55.0 \\
Shadowgraph technique [12] & 0.20 & $48.9-66.4$ \\
Otheriment & Hip simulator [13] & 0.15 & 13.0 \\
Current algorithm & Numerical method [20] & 0.11 & 41.5 \\
& FEM, no model modification & 0.109 & 42.0 \\
\hline
\end{tabular}

The diameter of the femoral head is $22 \mathrm{~mm}$.

Table 3

Comparison of wear depths/volumes of acetabular cup obtained from clinical measurements, experimental procedures and numerical methods

\begin{tabular}{llll}
\hline Algorithm & Technique/model & Wear depth (mm/year) & Wear volume (mm ${ }^{3} /$ year) \\
\hline Clinical measurement & Radiograph technique [10] & $0-0.3$ (mean: 0.08) & $0-225.0$ (mean: 48.4) \\
Others & Numerical method [20] & 0.111 & 16.0 \\
Current algorithm & FEM, with model modification & 0.085 & 52.072 \\
\hline
\end{tabular}

The diameter of the femoral head is $28 \mathrm{~mm}$. 
Table 4

Comparison of wear depths/volumes of acetabular cup obtained from clinical measurements, experimental procedures and numerical methods

\begin{tabular}{lllc}
\hline Algorithm & Technique/model & Wear depth (mm/year) & Wear volume (mm ${ }^{3} /$ year) \\
\hline Clinical measurement & Radiograph technique [10] & $0-0.32$ (mean: 0.10) & $3.0-256.0(\mathrm{mean}: 84.1)$ \\
Experiment & Hip simulator [13] & & $155.3-225.5$ \\
Others & Numerical method [20] & 0.116 & 18.0 \\
Current algorithm & FEM, with model modification & 0.073 & 58.417 \\
\hline
\end{tabular}

The diameter of the femoral head is $32 \mathrm{~mm}$.

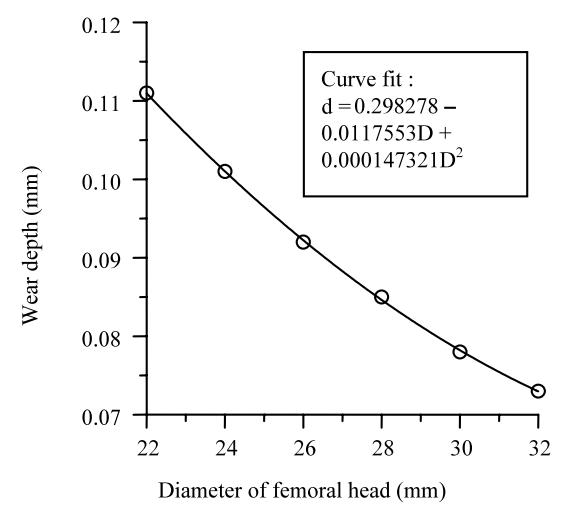

Fig. 10. Wear depth (d) versus diameter of femoral head (D).

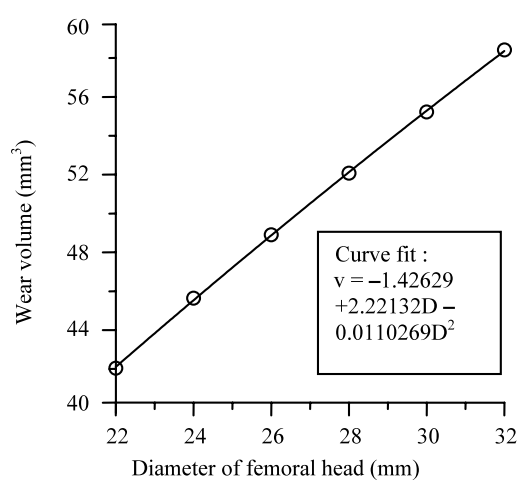

Fig. 11. Wear volume (v) versus diameter of femoral head (D).

yearly wear depth is 0.111 (mm per year) and the average wear volume is $42\left(\mathrm{~mm}^{3}\right.$ per year), which are very close to the results obtained from clinical measurements and experimental data. On basis of the results obtained here, it is easy for us to understand the wear rate on the polyethylene acetabular cup in an artificial hip joint under
Table 5

Wear depths/volumes of acetabular cup for different sizes of femoral head obtained using the current algorithm

\begin{tabular}{lll}
\hline $\begin{array}{l}\text { Diameter of femoral } \\
\text { head }(\mathrm{mm})\end{array}$ & $\begin{array}{l}\text { Wear depth } \\
(\mathrm{mm} / \text { year })\end{array}$ & $\begin{array}{l}\text { Wear volume } \\
\left(\mathrm{mm}^{3} / \text { year }\right)\end{array}$ \\
\hline 22 & 0.111 & 42.040 \\
24 & 0.101 & 45.639 \\
26 & 0.092 & 48.901 \\
28 & 0.085 & 52.072 \\
30 & 0.078 & 55.224 \\
32 & 0.073 & 58.417 \\
\hline
\end{tabular}

normal walking activity. Wear depths/volumes of the pin are correlated closely with the contact area and the maximum contact Von Mises stress is well below the Von Mises yielding strength for a polymer pin; so, plastic strains are hard to produce at the counterface and the occurrence of fatigue wear is impossible. Results of the study also suggest that the larger femoral head may induce larger wear volume, but gives a smaller wear depth and that wear depth and volume loss are apparently nonlinear related to the femoral head diameter. It can be said that the computer simulation algorithm proposed here yields realistic results and is clearly feasible. The current algorithms are useful and helpful in understanding wear behavior for alternative or new designs of artificial hip joints and even for other analogous structures. Furthermore, wear behavior of the artificial hip joints under impact events caused by exercise or accident may also be explored based on algorithms proposed here, which will be reported in other issues. 


\section{Acknowledgements}

This work was supported by the National Science Council, Taiwan, ROC.

\section{References}

[1] H. Willert, H. Bertram, G. Buchhorn, Osteolysis in alloarthroplasty of the hip, the role of ultra-high molecular weight polyethylene wear particle, Clin. Orthop. Relat. R 258 (1990) 95-107.

[2] H. Willert, G. Semlitsch, Reactions of articular capsule to wear products of artificial joint prostheses, Biomed. Mater. Res. 11 (1997) 157-164.

[3] T.P. Schmalzried, M. Jasty, W.H. Harris, Periprosthetic bone loss in total hip arthroplasty, J. Bone Joint Surg. 74A (1994) 849-863.

[4] D.W. Howie, B.L. Cornish, B. Vernon-Roberts, Resurfacing hip arthroplasty. Classification of loosening and the role of prosthetic wear particles, Clin. Orthop. 255 (1990) 144-159.

[5] J.K. Maguire, M.F. Coscia, M.H. Lynch, Foreign body reaction to polymeric debris following total hip arthroplasty, Clin. Orthop. 216 (1987) 213-223.

[6] H.M. Schuller, R.K. Marti, Ten-year socket wear in 66 hip arthoplastic ceramic versus metal heads, Acta Orthop. Scand. 61 (3) (1990) 240-243.

[7] B.M. Wroblewski, Wear and loosening of socket in the charnley low-friction arthoplasty, Clin. Orthop. North Am. 19 (1988) 627-630.

[8] J. Charnley, D.K. Halley, Rate of wear in total hip replacement, Clin. Orthop. 112 (1975) 170-179.

[9] J.R. Atkinson, D. Dowson, J.H. Isaac, B.M. Wroblewski, Laboratory wear tests and clinical observations of the penetration of femoral heads into acetabular cups in total replacement hip joints, Wear 104 (1985) 225-244.

[10] J. Livermore, D. Ilstrup, B. Morrey, Effect of femoral head size on wear of the polyethylene acetabular component, J. Bone Joint Surg. $72-4$ (1990) 518-528.

[11] J.M. Kabo, J.S. Gebhard, G. Loren, et al., In vivo wear of polyethylene acetabular components, J. Bone Joint Surg. (Br) 75 (1992) 497-504.

[12] R.M. Hall, A. Unsworth, P. Siney, B.M. Wroblewski, Wear in retrieved charnley acetabular sockets, Proc. Instn. Mech. Eng. 210 (1996) 197-207.

[13] J.H. Chen, J.S.S. Wu, Measurement of polyethylene wear-A three-dimensional methodology, Comput. Meth.
Prog. Biomed. 2001 (to be published in Computer Methods and Programs in Biomedicine).

[14] V. Saikko, P. Paavolainen, M. Kleimola, P. Slatis, A five-station hip joint simulator for wear rate studies, Proc. Instn. Mech. Eng. 206 (1992) 195-200.

[15] V. Saikko, Three-axis hip joint simulator for wear and friction studies on total hip prostheses, Proc. Instn. Mech. Eng. 210 (1996) 175-185.

[16] A. Wang, D.C. Sun, S.S. Yau, et al., Orientation softening in the deformation and wear of ultra-high molecular weigh polyethylene, Wear 203 (1997) 230-241.

[17] A. Wang, C. Stark, J.H. Dumbleton, Mechanistic and morphological origins of ultra-high molecular weight polyethylene wear debris in total joint replacement prostheses, Proc. Instn. Mech. Eng. 210 (1996) 141-155.

[18] R. Pietrabissa, M. Rainmondi, E.D. Martino, Wear of polyethylene cups in total hip arthoplasty: a parametric mathematical model, Med. Eng. Phys. 20 (1998) 199-210.

[19] Z.M. Jin, D. Dowson, J. Fisher, A parametric analysis of the contact stress in ultra-high molecular weight polyethylene acetabular cups, Med. Eng. Phys. 16 (1994) 398-405.

[20] T.A. Maxian, T.D. Brown, D.R. Pedersen, et al., A sliding-distance-couple finite element formulation for polyethylene wear in total hip arthroplasty, J. Biomech. 29-5 (1996) 687-692.

[21] A. Wang, A. Essner, V.K. Polineni, et al., Lubrication and wear of ultra-high molecular weight polyethylene in total joint replacements, Tribol. Int. 21 (1-3) (1998) 17-33.

[22] Y.Q. Wang, J. Li, Sliding wear behavior and mechanism of ultra-high molecular weight polyethylene, Mater. Sci. Eng. 266A (1999) 155-160.

[23] H.C. Meng, K.C. Ludema, Wear model and predictive equations: their form and content, Wear 181-183 (1995) 443-457.

[24] G. Beer, An isoparametric joint/interface element for finite element analysis, Int. J. Num. Method Eng. 21 (1985) 585-600.

[25] V. Saikko, A multidirectional motion pin-on-disk wear test method for prosthetic joint materials, J. Biomed. Mater. Res. 41 (1) (1998) 58-64.

[26] D. Dowson, N.C. Wallbridge, Laboratory wear tests and clinical observations of the penetration of femoral heads into acetabular cups in total replacement hip joints, Wear 104 (1985) 203-215.

[27] P.S.M. Barbour, D.C. Barton, J. Fisher, The influence of contact stress on the wear of UHMWPE for total replacement hip prostheses, Wear 181-183 (1995) 250-257. 